University of Wollongong

Research Online

Faculty of Engineering - Papers (Archive)

Faculty of Engineering and Information

Sciences

$1-1-2011$

\title{
Giant magnetic flux jumps in single crystals of $\mathrm{Ba} 0.6 \mathrm{~K} 0.4 \mathrm{Fe} 2 \mathrm{As} 2$
}

Ki-Young Choi

Sogang University Seoul

Gun Sang Jeon

Ewah Womans University Seoul Republic of Korea

X.F Wang

University of Science and Technology China

X.H Chen

Hefei National Laboratory China

Xiaolin Wang

University of Wollongong, xiaolin@uow.edu.au

See next page for additional authors

Follow this and additional works at: https://ro.uow.edu.au/engpapers

Part of the Engineering Commons

https://ro.uow.edu.au/engpapers/4071

\section{Recommended Citation}

Choi, Ki-Young; Jeon, Gun Sang; Wang, X.F; Chen, X.H; Wang, Xiaolin; Jung, Myung-Hwa; Lee, Sung-lk; and Park, Gangseo: Giant magnetic flux jumps in single crystals of Ba0.6K0.4Fe2As2 2011, 1-3.

https://ro.uow.edu.au/engpapers/4071

Research Online is the open access institutional repository for the University of Wollongong. For further information contact the UOW Library: research-pubs@uow.edu.au 


\section{Authors}

Ki-Young Choi, Gun Sang Jeon, X.F Wang, X.H Chen, Xiaolin Wang, Myung-Hwa Jung, Sung-lk Lee, and Gangseo Park 


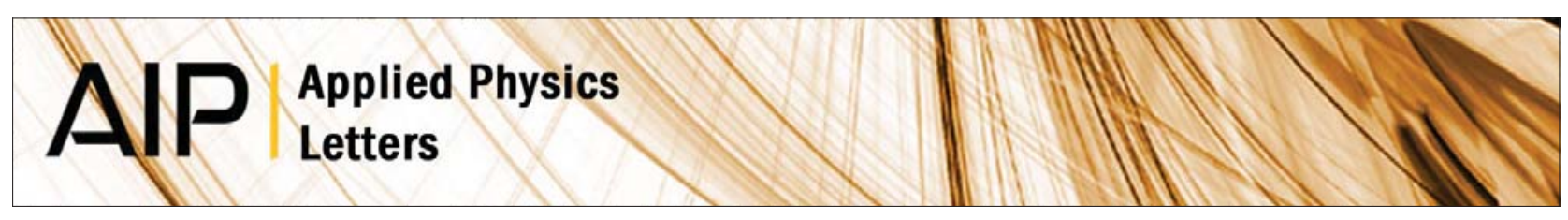

\section{Giant magnetic flux jumps in single crystals of Ba0.6K0.4Fe2As2}

Ki-Young Choi, Gun Sang Jeon, X. F. Wang, X. H. Chen, Xiao-Lin Wang et al.

Citation: Appl. Phys. Lett. 98, 182505 (2011); doi: 10.1063/1.3586777

View online: http://dx.doi.org/10.1063/1.3586777

View Table of Contents: http://apl.aip.org/resource/1/APPLAB/v98/i18

Published by the American Institute of Physics.

\section{Related Articles}

Superconductivity at $5 \mathrm{~K}$ in NdO0.5F0.5BiS2

J. Appl. Phys. 113, 056102 (2013)

Observation of partial Meissner effect and flux pinning in superconducting lead containing non-superconducting parts

Appl. Phys. Lett. 101, 162603 (2012)

Superconducting epitaxial thin films of $\mathrm{CeNixBi2}$ with a bismuth square net structure Appl. Phys. Lett. 101, 162602 (2012)

Superconductivity and magnetism in the $\mathrm{KxMoO} 2-\delta$

J. Appl. Phys. 112, 073923 (2012)

Magnetic pinning effects of epitaxial LaxSr1-xMnO3 nanostructured thin films on YBa2Cu3O7- $\delta$ layers J. Appl. Phys. 112, 053919 (2012)

\section{Additional information on Appl. Phys. Lett.}

Journal Homepage: http://apl.aip.org/

Journal Information: http://apl.aip.org/about/about_the_journal

Top downloads: http://apl.aip.org/features/most_downloaded

Information for Authors: http://apl.aip.org/authors

\section{ADVERTISEMENT}

\section{MP Applied Physics Letters}

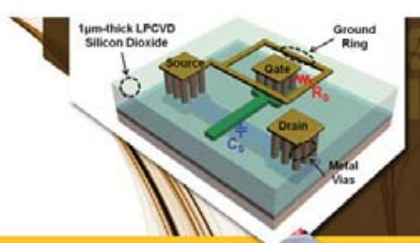

SURFACES AND INTERFACES

Focusing on physical, chemical, biological, structural, optical, magnetic and electrical interfaces, and more...

\section{EXPLORE WHAT'S NEW IN APL}




\title{
Giant magnetic flux jumps in single crystals of $\mathrm{Ba}_{0.6} \mathrm{~K}_{0.4} \mathrm{Fe}_{2} \mathrm{As}_{2}$
}

\author{
Ki-Young Choi, ${ }^{1, a)}$ Gun Sang Jeon, ${ }^{3}$ X. F. Wang, ${ }^{4}$ X. H. Chen, ${ }^{4}$ Xiao-Lin Wang, ${ }^{5}$ \\ Myung-Hwa Jung, ${ }^{2}$ Sung-lk Lee, ${ }^{2}$ and Gangseo Park ${ }^{2, a}$ \\ ${ }^{1}$ FPRD \& CeNSCMR, Department of Physics and Astronomy, Seoul National University, Seoul 151-747, \\ Republic of Korea \\ ${ }^{2}$ Department of Physics, Sogang University, Seoul 121-742, Republic of Korea \\ ${ }^{3}$ Department of Physics, Ewha Womans University, Seoul 120-750, Republic of Korea \\ ${ }^{4}$ Department of Physics, Hefei National Laboratory for Physical Sciences at the Microscale, \\ University of Science and Technology of China, Hefei, Anhui 230026, People's Republic of China \\ ${ }^{5}$ Institute for Superconducting and Electronic Materials, University of Wollongong, Wollongong, \\ New South Wales 2522, Australia
}

(Received 10 March 2011; accepted 13 April 2011; published online 5 May 2011)

\begin{abstract}
Giant magnetic flux jumps are observed in magnetic hysteresis loops of $\mathrm{Ba}_{0.6} \mathrm{~K}_{0.4} \mathrm{Fe}_{2} \mathrm{As}_{2}$ single crystals. The size of the flux jumps, which appear only at low temperatures $(T<4 \mathrm{~K})$, is so large that it can transform the whole superconducting state into the normal state. The recovery rate to the superconducting state is rather slow, although the superconducting state is almost fully recovered. We show that theoretical predictions based on the adiabatic approach with nonlocal electrodynamics give a good explanation of the flux jumps observed in the single crystals of $\mathrm{Ba}_{0.6} \mathrm{~K}_{0.4} \mathrm{Fe}_{2} \mathrm{As}_{2}$. (C) 2011 American Institute of Physics. [doi:10.1063/1.3586777]
\end{abstract}

Flux jumps in superconductors are quite an interesting phenomenon and have been observed in thin films of many superconductors, such as $\mathrm{MgB}_{2}{ }^{1,2} \mathrm{Nb},{ }^{3} \mathrm{Nb}$ alloys, $\mathrm{YNi}_{2} \mathrm{~B}_{2} \mathrm{C}$, ${ }^{5}$ and $\mathrm{YBa}_{2} \mathrm{Cu}_{3} \mathrm{O}_{7-\mathrm{x}} \cdot{ }^{6}$. Direct observation of flux jumps by magneto-optical imaging shows magnetic avalanches in thin films with dendritic or fingerlike patterns on a macroscopic scale inside the superconductors. Mostly, this phenomenon appears in the low field and low-temperature regime in the superconducting state. At high temperatures and high magnetic fields, vortex penetration occurs rather gradually, and vortex avalanches are not expected. The origin of this phenomenon is known to be the thermomagnetic instability which is induced by the positive feedback effect of vortex penetration into the superconductor combined with the sudden redistribution of the Abrikosov vortices inside the superconductor. ${ }^{1}$ In particular, when the diffusion of magnetic flux is much higher than the diffusion of heat, a superconductor becomes thermally unstable. Ironically, its high critical current density generates substantial amounts of Joule heat by the fast invasion of the vortices and produces the local normal path which subsequently induces the penetration of the vortices in the form of a vortex jump. Although flux jumps were reported to exist in $\mathrm{Bi}_{2} \mathrm{Sr}_{2} \mathrm{CaCu}_{2} \mathrm{O}_{8+\delta},{ }^{7}$ the occurrence of the vortex jumps is still very rare in polycrystalline superconductors. It was reported that the flux jumps were observed in the special case of $\mathrm{YNi}_{2} \mathrm{~B}_{2} \mathrm{C}$ single crystals, ${ }^{8}$ in which the flux jump was accompanied by a change in the local symmetry of the vortex lattice from low field rhombohedral to high field rhombohedral. This sudden change in the vortex lattice symmetry might be accompanied by local heating, thus triggering the flux jump. In this sense, the origin of the flux jumps in $\mathrm{YNi}_{2} \mathrm{~B}_{2} \mathrm{C}$ single crystals may not be exactly the same as that of the vortex jumps in other types of superconducting thin films.

\footnotetext{
${ }^{a)}$ Electronic addresses: kychoikc@snu.ac.kr and gpark@sogang.ac.kr.
}

In this letter, we report the observation of giant flux jumps in an optimally doped $\mathrm{Ba}_{0.6} \mathrm{~K}_{0.4} \mathrm{Fe}_{2} \mathrm{As}_{2}$ single crystal, which is the only compound that exhibits the flux jumps in single crystals, ${ }^{9}$ due to its very high intrinsic flux pinning strength, except for the special case of $\mathrm{YNi}_{2} \mathrm{~B}_{2} \mathrm{C}$. We studied single crystals of $\mathrm{Ba}_{0.6} \mathrm{~K}_{0.4} \mathrm{Fe}_{2} \mathrm{As}_{2}$ at temperatures below $4 \mathrm{~K}$. We find that flux jumps appear more closely spaced as the temperature becomes lower and the field sweep rate becomes higher in $M-H$ loops. We further observe that once a flux jump happens, this phenomenon occurs very fast. Also, the size of the flux jumps is large enough to drive the whole superconducting state in a single crystal into the normal state. In this respect, the flux jumps observed in this work differ from those in $\mathrm{Ba}_{0.72} \mathrm{~K}_{0.28} \mathrm{Fe}_{2} \mathrm{As}_{2}$ crystal ${ }^{9}$ with flux jumps observed at $T=5 \mathrm{~K}$ and those in the superconducting thin films, in which vortex invasion is very limited in the sample. We also find that the recovery rate from a flux jump is very slow, which indicates that the vortices invading the superconductor resist being expelled until $M-H$ curve has almost fully recovered to the superconducting state.

The single crystals of $\mathrm{Ba}_{0.6} \mathrm{~K}_{0.4} \mathrm{Fe}_{2} \mathrm{As}_{2}$ were prepared by the high-temperature self-flux method. The detailed procedure of crystal growth is described in Ref. 10. The superconducting transition temperature, $T_{c}$, of the single crystal was $37 \mathrm{~K}$. The dimensions of the sample were $2.0 \times 1.5$ $\times 0.12 \mathrm{~mm}^{3}$. The $M-H$ loops were collected with a field sweep rate of 1-70 mT/s by using a superconducting quantum interference device-vibrating sample magnetometer (SQUID-VSM, Quantum Design) with the applied field parallel to the $c$ axis $(H \| c)$ between -7 and $7 \mathrm{~T}$.

Figure 1 shows the magnetization hysteresis loops measured at different temperatures for the field sweep rate of 5 $\mathrm{mT} / \mathrm{s}$ for fields from -7 to $7 \mathrm{~T}$. We draw magnetization loops up to the fifth quadrant curves, since the first quadrant curves usually show fewer flux jumps due to the barrier against the first jump near the lower critical field $H_{c 1}$. The full hysteresis loop at $2 \mathrm{~K}$ shows four large-scale flux jumps, while no flux jumps are observed at $4.5 \mathrm{~K}$, and only one flux jump is 


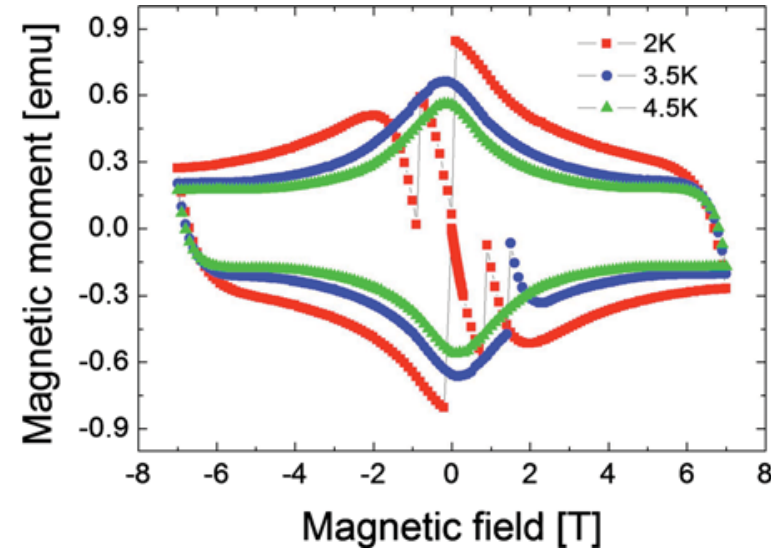

FIG. 1. (Color online) Magnetization hysteresis loops at 2, 3.5, and $4 \mathrm{~K}$ at a field sweep rate of $5 \mathrm{mT} / \mathrm{s}$.

observed at $3.5 \mathrm{~K}$, which implies that the flux jumps are limited to low temperature. We note that the field of the first flux jump $\left(H_{f j}\right)$ at $2 \mathrm{~K}$ is around $1 \mathrm{~T}$, which is orders of magnitude larger compared to the case of thin films. This implies that it is very difficult to achieve the instability condition in a single crystal specimen.

In addition to the flux jumps at such a large field of $\sim 1 \mathrm{~T}$, it is also surprising to observe the large size of the flux jumps in this single crystal. As seen in Fig. 1, each flux jump drives the entire superconducting state into the normal state with $M=0$. This is in sharp contrast with the flux jumps in the superconducting thin films, in which vortex invasion is very limited. Our observations show that this phenomenon occurs so fast that the SQUID-VSM can pick up the data only before and after the flux jump, even at the fastest sweep rate $(\sim 70 \mathrm{mT} / \mathrm{s})$ in our measurements. However, the recovery of the superconducting state from the $M=0$ state is quite slow, but once it has recovered, invading flux is almost fully expelled. This has never been observed in superconducting thin films.

Figure 2 shows the $M-H$ curves at $2 \mathrm{~K}$ with a sweep rate of $5 \mathrm{mT} / \mathrm{s}$ for two different measurements. The two measured $M-H$ curves are different; the first measurement shows four flux jumps while the second shows only two flux jumps. This implies that flux jumping is a stochastic phenomenon which shows different patterns at each measurement for the same sample under the same external conditions.

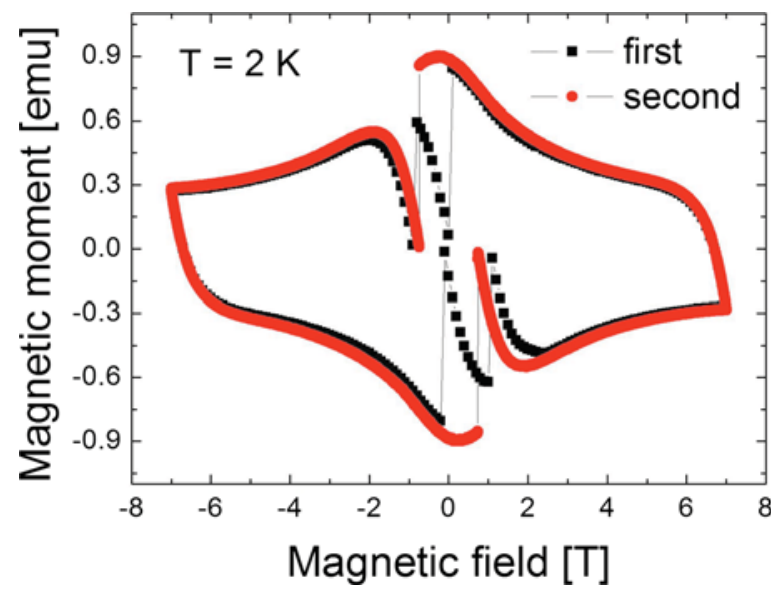

FIG. 2. (Color online) Magnetization hysteresis curves measured at $2 \mathrm{~K}$ with a sweep rate of $5 \mathrm{mT} / \mathrm{s}$ for two different measurements.

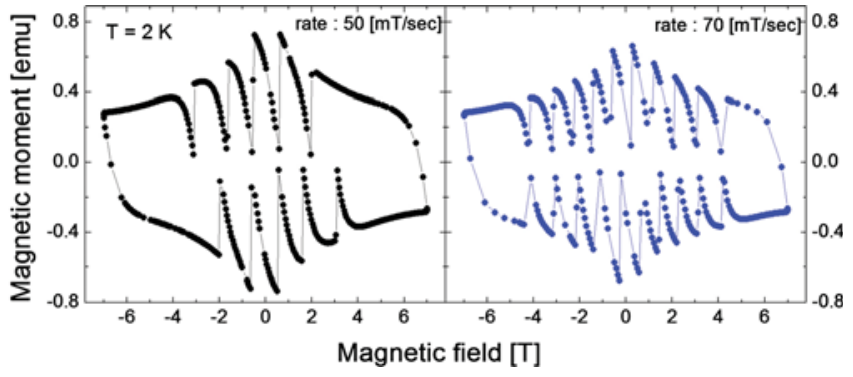

FIG. 3. (Color online) Magnetization hysteresis loops at $2 \mathrm{~K}$ for the field sweep rates of $50 \mathrm{mT} / \mathrm{s}$ and $70 \mathrm{mT} / \mathrm{s}$, respectively.

We also increased the field sweep rate up to $70 \mathrm{mT} / \mathrm{s}$. Figure 3 shows that different sweep rates of the magnetization hysteresis at the same temperature result in different patterns of flux jumps. As the field sweep rate is increased, the activity of the flux jumps becomes enhanced, and the flux jumps appear more closely spaced.

The flux jump is an avalanche phenomenon that takes place when the first penetration of vortices induces subsequent vortex motion. It occurs when the magnetic diffusion rate is faster than the thermal diffusion rate in the superconductor. ${ }^{11}$ When the applied field is increased to a certain critical value, the Lorentz force at a local point inside the superconductor exceeds the vortex pinning force, and lines of magnetic vortices move suddenly into the superconductor, thus triggering a flux jump. This flux jump does not proceed when the energy dissipated by the flux jump is rapidly absorbed by the superconducting sample. In our single crystals, the energy dissipated is so large that the full superconducting state can be driven into the normal state.

The question then arises whether magnetic instability of the $\mathrm{Ba}_{0.6} \mathrm{~K}_{0.4} \mathrm{Fe}_{2} \mathrm{As}_{2}$ crystal has the same origin as in the thin films or not. Since the thickness of our sample used in this experiment is much smaller than the width of the sample, we treat our sample in the thin film limit and apply the formula based on the adiabatic approach with nonlocal electrodynamics. ${ }^{12,13}$ The first flux jump field, $H_{f j}$, from thermomagnetic origin, is explained as follows: ${ }^{13}$

$$
\begin{aligned}
& H_{f j}=\frac{J_{c} d}{\pi} \cosh ^{-1}\left(\frac{w}{w-l^{*}}\right), \\
& l^{*}=\frac{\pi}{2} \sqrt{\frac{\kappa}{\left|J_{c}^{\prime}\right| E}}\left(1-\sqrt{\frac{2 h_{0}}{n d\left|J_{c}^{\prime}\right| E}}\right)^{-1} .
\end{aligned}
$$

Here, we assume that the thickness $d$ is much smaller than half of the sample width $w$. The parameter $n \gg 1$ implies a strongly nonlinear current-voltage characteristic of the superconductor with the relation of $E \propto J^{n}$. We set $n$ to be 30, which is a commonly used value. For our sample we use the thermal conductivity, $\kappa$, at $2 \mathrm{~K}$ of $0.45 \mathrm{~W} / \mathrm{Km} .{ }^{14}$ The temperature dependence of the critical current density is given by $J_{c}(T)=J_{c 0}\left(1-T / T_{c}\right)$, where we estimate $J_{c 0}$ as 8 $\times 10^{9} \mathrm{~A} / \mathrm{m}^{2}$ from Bean's critical state model. The heat transfer coefficient to the surrounding environment, $h_{0}$, is taken to be $10^{4}\left(T / T_{c}\right)^{3} \mathrm{~W} / \mathrm{K} \mathrm{m}^{2} .^{15}$ The induced electric field $E$ in the formula is fitted to our experimental results, yielding $E=2.2(1.8) \mathrm{mV} / \mathrm{m}$ at the field sweep rate of $70(50) \mathrm{mT} / \mathrm{s}$, under the assumption that a fast sweep rate generates a higher electric field. Using the above parameters, we obtain $H_{f j}$ as $0.83 \mathrm{~T}$ at $70 \mathrm{mT} / \mathrm{s}$ and $1.02 \mathrm{~T}$ at $50 \mathrm{mT} / \mathrm{s}$, which is in 
good agreement with experimentally observed first jump fields, $0.84 \mathrm{~T}(1.03 \mathrm{~T})$ at $70 \mathrm{mT} / \mathrm{s}(50 \mathrm{mT} / \mathrm{s})$.

Such a good agreement reveals that the magnetic flux jumps observed in our $\mathrm{Ba}_{0.6} \mathrm{~K}_{0.4} \mathrm{Fe}_{2} \mathrm{As}_{2}$ single crystal belong to the same class as the vortex jumps that are mostly observed in thin films, even though the $H_{f j}$ values are orders of magnitude different. The high value of $H_{f j}$ in the single crystal can be also explained by the geometric effect due to the larger thickness in a single crystal. Since $d$ is much larger in the single crystals than in the thin films, we can see from Eq. (2) that $H_{f j}$ in the single crystals becomes much larger than in the thin films.

In conclusion, we have observed giant magnetic flux jumps in single crystal $\mathrm{Ba}_{0.6} \mathrm{~K}_{0.4} \mathrm{Fe}_{2} \mathrm{As}_{2}$ superconductor. The flux jumps appear at low temperatures below $4 \mathrm{~K}$. The number of flux jumps depends on the sweep rate, and they occur more frequently at a higher field sweep rate. The size of the flux jumps is large enough that the whole superconducting state can be transformed into the normal state. The recovery rate of the superconducting state is quite slow, but once it has recovered, the invading flux vortices are almost fully expelled outside the superconductor. The critical state model based on the adiabatic approach with nonlocal electrodynamics was applied to explain this magnetic instability.

We gratefully acknowledge helpful discussions with Kee Hoon Kim. This work was supported by the National Creative Research Initiative (Grant No. 2010-0018300) and the Special Fund of Sogang University. We thank the A3 foreign program for initiating this project. This work is also supported in part by the Ministry of Education, Science and Technology/National Research Foundation (MEST/NRF) of Korea (Grant No. 2010-0010937) and the Australian Research Council through Discovery projects (Grant Nos.
DP0558753 and DP1094037).

${ }^{1}$ T. H. Johansen, M. Baziljevich, D. V. Shantsev, P. E. Goa, Y. M. Galperin, W. N. Kang, H. J. Kim, E. M. Choi, M.-S. Kim, and S. I. Lee, Europhys. Lett. 59, 599 (2002)

${ }^{2}$ Z. W. Zhao, S. L. Li, Y. M. Ni, H. P. Yang, Z. Y. Liu, H. H. Wen, W. N. Kang, H. J. Kim, E. M. Choi, and S. I. Lee, Phys. Rev. B 65, 064512 (2002).

${ }^{3}$ I. A. Rudnev, D. V. Shantsev, T. H. Johansen, and A. E. Primenko, Appl. Phys. Lett. 87, 042502 (2005).

${ }^{4}$ I. A. Rudnev, S. V. Antonenko, D. V. Shantsev, T. H. Johansen, and A. E. Primenko, Cryogenics 43, 663 (2003).

${ }^{5}$ S. C. Wimbush, B. Holzapfel, and C. Jooss, J. Appl. Phys. 96, 3589 (2004).

${ }^{6}$ P. Leiderer, J. Boneberg, P. Brüll, V. Bujok, and S. Herminghaus, Phys. Rev. Lett. 71, 2646 (1993).

${ }^{7}$ A. Nabiałek, M. Niewczas, H. Dabkowska, A. Dabkowski, J. P. Castellan, and B. D. Gaulin, Phys. Rev. B 67, 024518 (2003).

${ }^{8}$ D. Jaiswal-Nagar, A. D. Thakur, S. Ramakrishnan, A. K. Grover, D. Pal, and H. Takeya, Phys. Rev. B 74, 184514 (2006).

${ }^{9}$ X.-L. Wang, S. R. Ghorbani, S.-I. Lee, S. X. Dou, C. T. Lin, T. H. Johansen, K.-H. Muller, Z. X. Cheng, G. Peleckis, M. Shabazi, A. J. Qviller, V. V. Yurchenko, G. L. Sun, and D. L. Sun, Phys. Rev. B 82, 024525 (2010).

${ }^{10}$ X. F. Wang, T. Wu, G. Wu, H. Chen, Y. L. Xie, J. J. Ying, Y. J. Yan, R. H. Liu, and X. H. Chen, Phys. Rev. Lett. 102, 117005 (2009).

${ }^{11}$ I. S. Aranson, A. Gurevich, M. S. Welling, R. J. Wijngaarden, V. K. Vlasko-Vlasov, V. M. Vinokur, and U. Welp, Phys. Rev. Lett. 94, 037002 (2005).

${ }^{12}$ D. V. Shantsev, A. V. Bobyl, Y. M. Galperin, T. H. Johansen, and S.-I. Lee, Phys. Rev. B 72, 024541 (2005).

${ }^{13}$ D. V. Denisov, D. V. Shantsev, Y. M. Galperin, E.-M. Choi, H.-S. Lee, S.-I. Lee, A. V. Boby, P. E. Goa, A. A. F. Olsen, and T. H. Johansen, Phys. Rev. Lett. 97, 077002 (2006).

${ }^{14}$ X. G. Luo, M. A. Tanatar, J.-P. Reid, H. Shakeripour, N. Doiron-Leyraud, N. Ni, S. L. Bud'ko, P. C. Canfield, H. Luo, Z. Wang, H.-H. Wen, R. Prozorov, and L. Taillefer, Phys. Rev. B 80, 140503 (2009).

${ }^{15}$ E. T. Swartz and R. O. Pohl, Rev. Mod. Phys. 61, 605 (1989). 